\title{
Enhancement of Dibenzothiophene Desulfurization by Gordonia alkanivorans Strain 1B Using Sugar Beet Molasses as Alternative Carbon Source
}

\author{
Luís Alves • Susana M. Paixão
}

Received: 29 November 2013 / Accepted: 27 January 2014 /

Published online: 12 February 2014

(C) Springer Science+Business Media New York 2014

\begin{abstract}
There are several problems limiting an industrial application of fossil fuel biodesulfurization, and one of them is the cost of culture media used to grow the microorganisms involved in the process. In this context, the utilization of alternative carbon sources resulting from agro-industrial by-products could be a strategy to reduce the investment in the operating expenses of a future industrial application. Recently, Gordonia alkanivorans 1B was described as a fructophilic desulfurizing bacterium, and this characteristic opens a new interest in alternative carbon sources rich in fructose. Thus, the goal of this study was to evaluate the utilization of sugar beet molasses (SBM) in the dibenzothiophene (DBT) desulfurization process using strain 1B. SBM firstly treated with $0.25 \% \mathrm{BaCl}_{2}(w / v)$ was used after sucrose acidic hydrolysis or in a simultaneous saccharification and fermentation process with a Zygosaccharomyces bailii Talf1 invertase (1\%), showing promising results. In optimal conditions, strain 1B presented a $\mu_{\max }$ of $0.0795 \mathrm{~h}^{-1}$, and all DBT was converted to 2hydroxybiphenyl $(250 \mu \mathrm{M})$ within $48 \mathrm{~h}$ with a maximum production rate of $7.78 \mu \mathrm{M} \mathrm{h}^{-1}$. Our results showed the high potential of SBM to be used in a future industrial fossil fuel biodesulfurization process using strain $1 \mathrm{~B}$.
\end{abstract}

Keywords Biodesulfurization - DBT - Sugar beet molasses · Gordonia alkanivorans strain 1B · SSF. Sulfate precipitation

\section{Introduction}

The technology used to reduce sulfur in fossil fuels consists of a process called hydrodesulfurization (HDS). This process is expensive since it is carried out at high temperature and pressure and does not work well on various polyaromatic sulfur heterocycles always present in crude oil such as dibenzothiophene (DBT) and substituted DBTs (methylated DBTs and benzo-DBTs), which are particularly recalcitrant to HDS [1]. To achieve lower sulfur

L. Alves $(\bowtie) \cdot$ S. M. Paixão $(\bowtie)$

LNEG - Laboratório Nacional de Energia e Geologia, IP, Unidade de Bioenergia,

Estrada do Paço do Lumiar, 22, 1649-7038 Lisbon, Portugal

e-mail: luis.alves@lneg.pt

e-mail: susana.alves@lneg.pt 